\title{
Research on Gesture Recognition Technology of Data Glove Based on Joint Algorithm
}

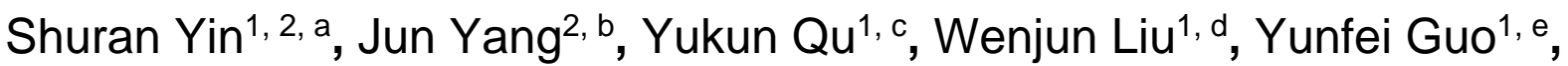 \\ Hongtao Liu ${ }^{1, \mathrm{f}}$, Dapeng Wei, ${ }^{1,2, \mathrm{~g}}{ }^{\text {* }}$ \\ ${ }^{1}$ School of Computer Science and Technology, Chongqing University of Posts and \\ Telecommunications, Chongqing 400065, China; \\ ${ }^{2}$ Chongqing Institute of Green and Intelligent Technology, Chinese Academy of Sciences, \\ Chongqing 400714, China.
}

asallyyinsr@qq.com, bjyang@cigit.ac.cn, cqyukun1992@163.com, d2662052636@qq.com, eguoyunfei.2007@163.com, fliuht@cqupt.edu.cn, ", g dpwei@cigit.ac.cn

Keywords: Gesture recognition, joint algorithm, data glove, bending sensor.

\begin{abstract}
A gesture recognition system were fabricated with distributed sensor networks as the core component, and could be wore in the hands. This data glove could detect the posture of fingers using the FLEX2.2 bending sensors and STM32 series single chip. Furthermore, we adopted a gesture recognition joint algorithm which combined template matching with BP neural network. First, the identify data from bending sensors could be obtained by template matching. Then, these data was inputted to the neural network, and got the final gesture recognition rate of joint algorithm. This joint algorithm enhanced minimal template recognition rate, and improved high training volume of BP neural network, showing the higher accuracy and adaptability of recognition. These research exhibit large potential in low cost, accurate, flexible, wearable sign language recognition systems.
\end{abstract}

\section{Introduction}

Gesture language is not only an important part of human language but also the branch of sign language. Compared with natural language, the use of gesture language can diversity our communication. The study of gesture recognition, on the one hand, can be used as the tools for hearing aid in work and life [1], especially helping hearing impaired people who are less educated use sign language to communicate with normal people; on the other hand, it can improve the computer's level of understanding and cognition of human language [2].

There are two main research methods for gesture recognition. One is visual image-based gesture recognition. The other is identification based on data glove-based. The former is built on computer vision, and obtains gesture image data through the camera, and then uses a variety of image processing technology to analyze the data, and finally realizes gesture recognition. The latter is through the data glove which is a wearable sensor device to capture the gesture of the original data, and then analyze the data to identify the user's hand shape [3]. The gesture recognition based on photographic images is natural and has good interaction effect. However, recognition devices are expensive, and the scene and environment of using the devices are also limited. It is very difficult to recognize the gesture in the complex environment [4]. But the gesture recognition technology based on data glove can send the user's gesture information to the recognition system without any problem [5]. Its equipment is simple and low cost, and it is not easily affected by the environment, and the accuracy and stability of the gesture recognition can be obtained to improve.

At present, gesture recognition technology mainly adopts template matching, an artificial neural network, hidden Markov model and Naive Bayesian classifier and other methods. The implementation principle of template matching is straightforward, but the variability of the template will greatly affect the recognition rate of gestures. Neural network method has the characteristics of anti-jamming and classification, fault-tolerant ability, less susceptible to noise interference, but easy to fall into the local minimum, slow convergence and other problems [6]. Hidden Markov model 
requires a large number of state probability density calculations, and needs to estimate a number of parameters. Identification and training speed is relatively slow, so the preceding gesture recognition use less hidden Markov model [7]. The naive Bayesian classifier is less used and has its flaws in terms of visual and data gloves, mainly in handwriting digital recognition [8].

Herein, this paper adopts a novel joint algorithm based on template matching and neural network. The algorithm solves the problem of low recognition rate of the template matching gesture recognition and reduces the training volume of neural network. By comparing the template matching algorithm, neural network algorithm and joint algorithm for different detectors gesture recognition rate, the results found that the joint algorithm in gesture recognition has a greater advantage.

\section{Gesture Collection}

\subsection{Data Glove Design.}

The gesture recognition based on data glove consists of five bending sensors attached to the joint of the finger and a circuit board attached to the sensor via the DuPont line. Bending sensor is a flexible sensor that can reflect the angle of finger bending. The change is translated into digital information through the lower computer to the host computer. Sensors are an important part of data gloves, affecting the accuracy of the collected data, so that affects the accuracy of gesture recognition. The sensor used in this paper is Spectra symbol flex2.2 "bending sensor, who's the initial resistance is about $25 \mathrm{~K} \Omega$, and in which the initial voltage is $0.5 \mathrm{~V}$. Resistance can be modified to $50 \mathrm{~K} \Omega$ after bending ninety degrees. When the metal face of the sensor is bent outward, the sensor resistance value will change. The serial port collects a change in voltage through the ADC, namely the gesture sample data.

It uses self-made single-chip circuit to collect the sensor data, through the USB serial cable to link the host computer and lower computer. The circuit board includes multiple selection switch (MUX), micro control unit (MCU), cp2102 (USB-UART bridge), AMP, power part, LED lights and other parts. As is shown in Figure 1, the figure is a bridge amplifier circuit of the data acquisition module. Bridge circuit can effectively inhibit the temperature drift. The magnification is:

$$
A=\frac{R_{7}}{R_{5}}=20
$$

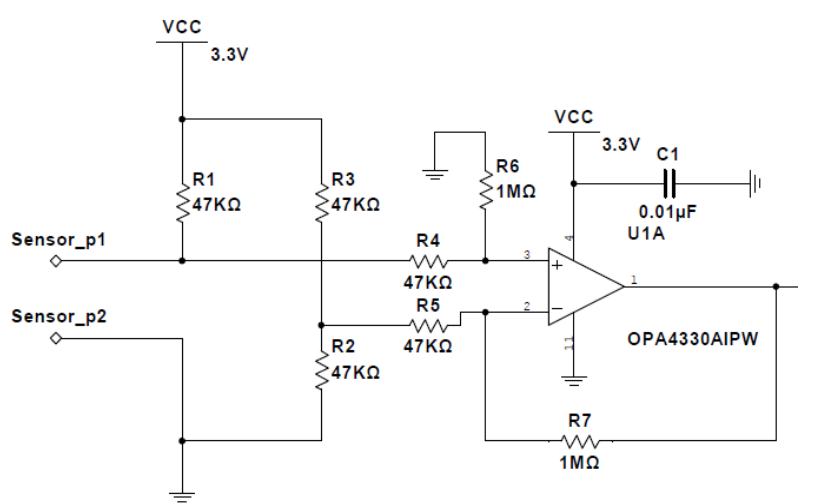

Fig. 1 Bridge amplifier circuit

The signal can be amplified to fit the need of $A / D$ conversion. Data glove sensor and microcontroller distribution are given in Figure 2.You can see the design of the microcontroller pin matches well with the two plugs of sensor. The connection of the DuPont line can prevent the line slip and break during the serial data acquisition [9]. The system block diagram is shown in Figure 3 . The tester puts on the data glove and exposes the gesture, and the sensor bending data is transmitted to the host computer through the data acquisition module on the data glove. After receiving this data, the host computer saves the data to the designated location. Then, analyze and deal with the data, such as noise processing, normalization, template creation, and the processed data will train for algorithm and get the recognition rate, finally, identify gestures and display them on the computer. 


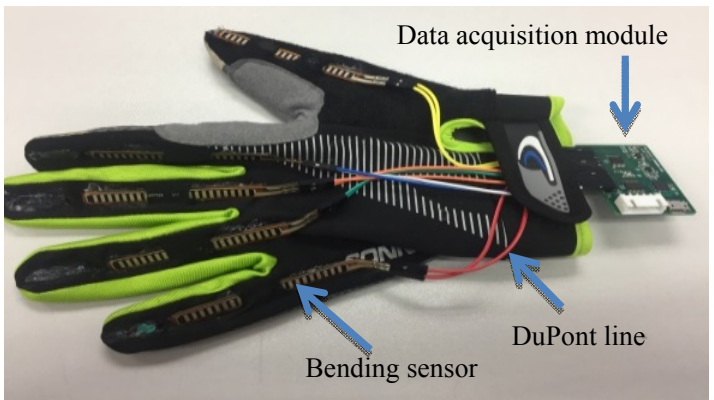

Fig. 2 Data glove appearance

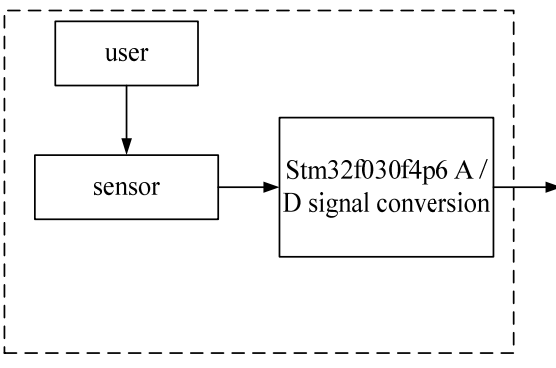

data acquisition module

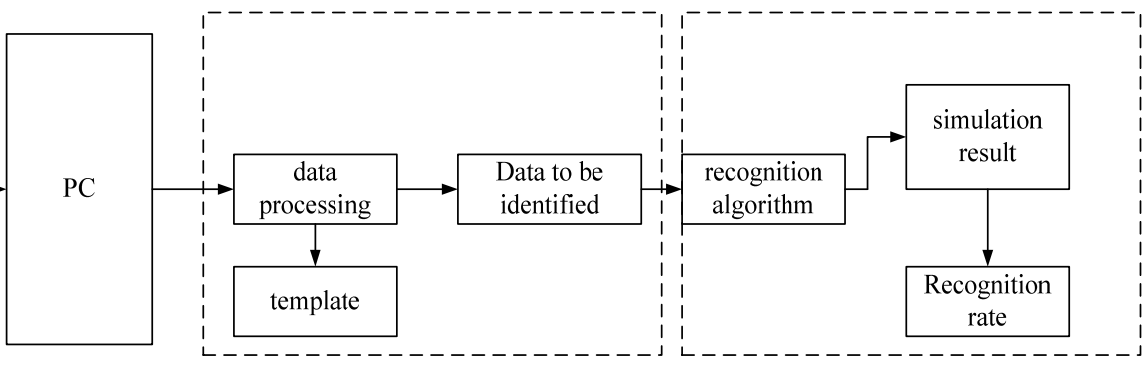

data processing module

Algorithm recognition module

Fig. 3 System block diagram

\subsection{Gesture Design.}

Gesture recognition can be subdivided into static gesture recognition and dynamic gesture recognition [10], in which dynamic gesture recognition can eventually be transformed into static gesture recognition. Static gesture recognition is the recognition of hand shape, which can be divided into digital gestures and alphabet gestures. However, dynamic gesture recognition is the recognition of opponent status, including daily communication language and vocabulary. In this paper, the gesture recognition is mainly on the recognition of static gestures, setting the intuitive distinction between the digital gestures 1-6 and alphabet gestures A, T, W. The specific gesture is shown in Figure 4.

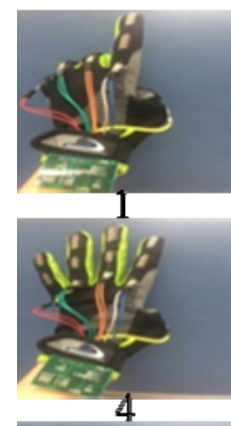

4

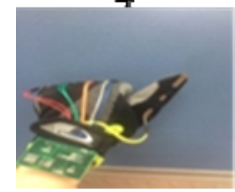

A
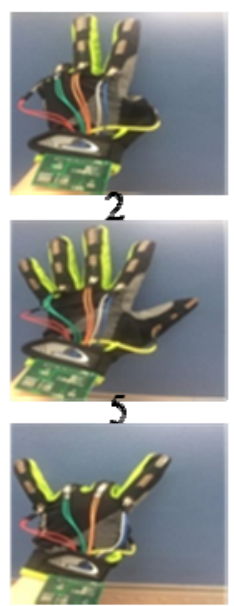

$\mathrm{T}$

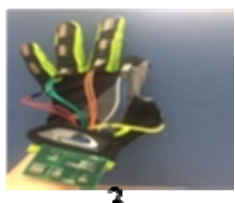

3
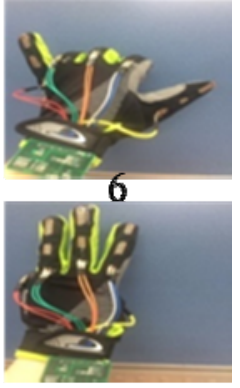

W

Fig. 4 Nine kinds of static gestures

\subsection{Gesture Acquisition Process.}

Gesture acquisition is the foundation of gesture recognition. We are required to train algorithms and create templates by collecting data. Gesture acquisition process is a process from the analog signal input to the data display process, based on the sensor and microcontroller design. In this paper, the lower computer uses the stm32 series of microcontrollers, which are based on ARM Cortex-M3 cores specifically designed for embedded applications requiring high performance, low cost, and low power [11]. It is suitable for hardware design and data transmission. 
This information acquisition program is written in accordance with SCM C language program in keil5. Because the data in this paper are mainly used to train the algorithm and establish a template, and there is no feedback, so there is no corresponding handshake mechanism.

In order to ensure the accuracy of data recognition, we collected the gestures of five people in different age and gender with the same gestures collected for 200 times, so that each gesture can get 1000 different data to ensure the training volume of the template matching and neural network.. The sample data in this paper includes nine gesture data, and each gesture data consists of five sensor values and one identification (which identifies each row of data being one of the gestures). Each gesture is collected for 1000 time, resulting in 9000 rows and 6 columns of data. Table 1 is a part of the sample data. The data represent voltage changes of bending the sensor when the thumb, index finger, middle finger, ring finger and little finger make the gesture of the sixth sign.

Table 1. Three Scheme comparing

\begin{tabular}{rccccc}
\hline Thumb & Index finger & Middle finger & Ring finger & Little finger & Gesture \\
\hline 1.565405 & 0.626807 & 0.639697 & 0.534155 & 0.700122 & 4 \\
1.144849 & 0.540601 & 1.266504 & 1.140820 & 0.710596 & $\mathrm{~T}$ \\
1.365601 & 0.683203 & 0.588940 & 0.495483 & 1.289868 & $\mathrm{~W}$ \\
1.489673 & 0.658228 & 1.155322 & 0.961157 & 1.294702 & 1 \\
0.520459 & 1.687866 & 1.060254 & 1.193188 & 1.447778 & $\mathrm{~A}$ \\
0.540601 & 0.592163 & 0.531738 & 0.468896 & 0.855615 & 5 \\
1.620190 & 1.645972 & 0.645337 & 0.439087 & 0.731543 & 3 \\
1.364795 & 0.586523 & 0.634863 & 1.104565 & 1.383325 & 2 \\
0.489844 & 1.789380 & 1.214136 & 1.037695 & 0.813721 & 6 \\
\hline
\end{tabular}

In the data acquisition process, the impact of noise is unavoidable. In view of this, in the designing of data acquisition module, it is required to select the appropriate low-noise op amp and amplifier circuit [12]. In this way, both the noise source can be suppressed and the output signal of the sensor is modulated to balance the amplitude modulation wave. Secondly, a low-pass filter can be adjusted on the circuit to filter out some signals exceeding the threshold value. As is shown in Figure 5 is the circuit noise suppression part of the circuit diagram, the left part is the frequency notch which interferences filter against $50 \mathrm{~Hz}$ power frequency; The right part is the band-pass filter, it consists of low-pass filter $\mathrm{fl}$ and high-pass filter fh in series, of which:

$$
\begin{gathered}
f_{l}=\frac{1}{(2 * 3.14 * R 16 * C 6)}=44.2 \mathrm{HZ} \\
f_{h}=\frac{1}{(2 * 3.14 * R 8 * C 2)}=0.08 \mathrm{HZ} \\
A_{u}=\frac{R_{15}}{R_{14}}=10
\end{gathered}
$$

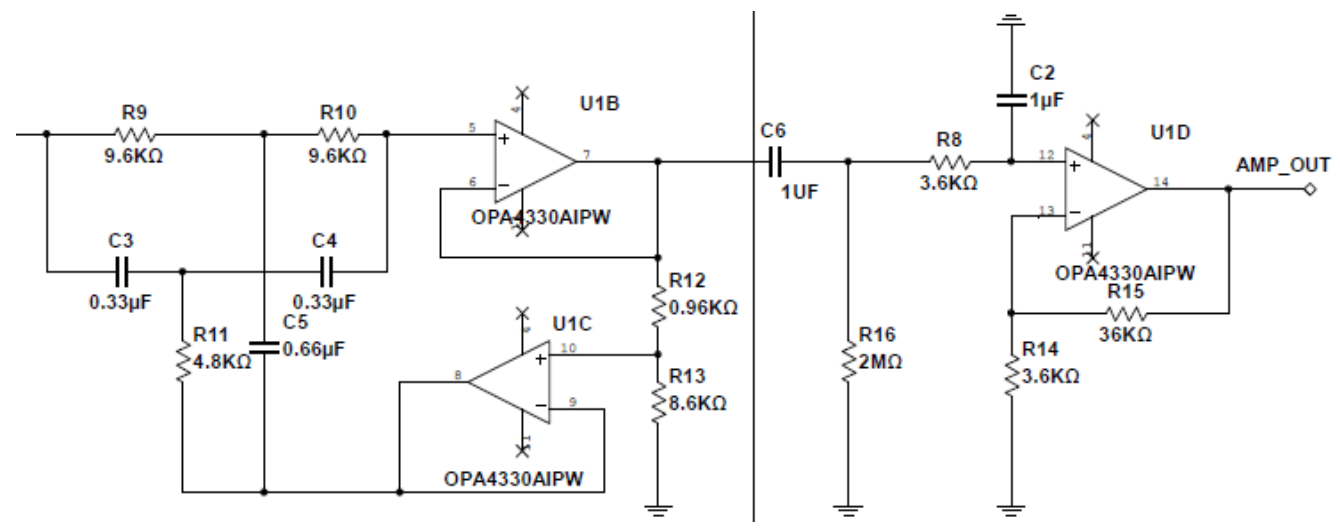

Fig. 5 Noise suppression circuit

You can see the magnification is 10 time, low-pass filter and high-pass filter is $0.08-44.2 \mathrm{HZ}$ and they can meet the design requirements, it will not affect the data. The above method can reduce the impact of noise on the recognition performance before data collection. 
In the meantime, noise suppression can be performed after data acquisition. Because the collected gesture data are based on the bending change of the bending sensor, there is not too much offset, and it will not fluctuate due to the interference of other electrical signals. Therefore, the principal component analysis can be used to analyze the collected data. This method can divide the data into independent components, remove unimportant and unimportant components and leave the core component to achieve noise reduction.

\section{Gesture Recognition Algorithm}

Built on the design of the data glove, this paper firstly uses two common algorithms, template matching algorithm and BP neural network algorithm (hereinafter referred to as BP algorithm) for gesture training recognition. However, due to the defects of the algorithm, such as easily-affected template matching and low recognition rate, neural network training is volume, and training time is too long, so that the existing identification algorithm is hard to get the best results. Therefore, this paper adopts a new joint algorithm of gesture recognition algorithm, a joint algorithm connected by template matching and BP algorithm. Experiments show that the joint algorithm can improve the recognition accuracy and shorten the training time.

\subsection{Template Matching.}

Template matching is a very generic pattern recognition method which was originally used for image processing. The template is known small images to search for. The template matching process is to search for a target in a large image according to the template size and direction to find the same image element [13]. As the template matching recognition is simple, and the principle is easy to understand, the algorithm is more and more utilized in the field of gesture recognition, and become the most basic gesture recognition method. Pattern matching of gesture recognition for data glove is to match the collected sensor data with pre-designed templates one by one, via measuring similarity to identify [14].

In this paper, the template base of gesture recognition for template matching is to average the per gesture of 1000 data for a single sensor (the value of each column of data), which is regarded as a template of the matching template. Then, the obtained sensor data is matched with the gesture template in the template base to calculate its Euclidean distance [14] (the following formula), that is, the data of each finger collected is $\mathrm{x} 1, \mathrm{x} 2 \ldots \mathrm{xn}$, the data of each finger of template is set to $\mathrm{y} 1, \mathrm{y} 2 \ldots \mathrm{yn}$. Each finger data is calculated separately from the template data, and then the $\mathrm{d}$ values are sorted to find the Euclidean minimum distance, and within the allowable range. It is recognized that the gesture is the user input gesture.

$$
d(x, y)=\sqrt{\sum_{i=1}^{n}\left(x_{i}-y_{i}\right)^{2}}
$$

\subsection{BP Neural Network.}

In this paper, a three-layer BP neural network is adopted, which includes an input unit, hidden layer and output layer [15]. The input unit has five neurons, corresponding to the data of the five sensors of data glove after the normalized value; the output layer has nine neurons, corresponding to the size of the probability that the sample belongs to a gesture. This paper implements the feed forward neural network established by MATLAB.

In the experiment, it needs to constantly adjust the number of hidden layer nodes, select different activation functions, and set separate learning rates. The number of hidden layer nodes has little effect on the recognition rate, but too many nodes will increase the number of computation, making the training slower. The neural network needs emphasize the activation function, and the activation function has a significant impact on the recognition rate or the convergence rate. Learning rate affects the speed of convergence of the network, and whether the network can converge. The small learning rate settings can ensure the convergence of the network, but the convergence rate is relatively slow. On the contrary, the large learning rate may make the network training not be converged and affect the recognition effect [15]. The parameters designed in this paper are reproduced below. The learning 
rate is 0.001 , the target error is less than 0.001 , the number of training is 2000 , and the training result is displayed every 50 time:

Net.trainparam. . how $=50$;

Net.trainparam.epochs $=2000$;

Net.trainparam.goal $=0.001$;

Net.trainparam.1r $=0.001$;

\subsection{Joint Algorithm.}

Although the above template matching and BP algorithm are commonly used in pattern recognition, because of the existence of the algorithm defects, such as the low template recognition rate which is largely affected by template and the long BP algorithm learning time, the final experimental accuracy and stability is not very accurate. This paper chooses a new joint algorithm that combines two algorithms [16].The identification process of joint algorithm is to firstly get the data to be input. This part of the data is considered to be identified after the template matching and Euclidean distance. This is considered to be a relatively high precision data, and then the identified data after normalization is as an input for BP algorithm, and get a higher recognition rate after training. The algorithm can be seen as experiencing two time recognition The first time is to obtain high-precision data; the second time, we make re-identification on the basis of high-precision data, and then further improve the accuracy of data.

The main description of the algorithm: Firstly, suppose the input gesture data is $X=\{x 1, x 2, \ldots$, $\mathrm{xn}\}$, the template to be matched is $\mathrm{Y}=\{\mathrm{y} 1, \mathrm{y} 2, \ldots, \mathrm{yn}\}$, the output gesture is $Z=\{\mathrm{z} 1, \mathrm{z} 2, \ldots, \mathrm{zn}\} . \mathrm{In}$ order To obtain the $\mathrm{Z}$ data from the $\mathrm{X}$ data, the minimum Euclidean distance between $\mathrm{X}$ and $\mathrm{Y}$ corresponding points needs to be calculated. That is:

$$
Z_{k}=\min \left(\sqrt{\sum_{i=1}^{n}\left(x_{i}-y_{i}\right)^{2}}\right)
$$

$\mathrm{Zk}$ is the value of the gesture represented by the minimum value, and the gesture data output of $\mathrm{Z}$ belonging to $\mathrm{Zk}$ is stored under the MATLAB document. At this point, 9000 gesture values can eventually identify 8707 data; Secondly, this data is normalized using the following formula:

$$
y=\frac{x-\min }{\max -\min }
$$

Where $\mathrm{x}$ is the input vector, that is, the reading of each sensor of the glove, max, min are the maximum and minimum readings of the sensor, $y$ is the output vector, that is, the value of the normalized sensor and the nerve Network input; Finally, the neural network model is established with 5 input neurons and 9 output neurons.

The gesture recognition flow chart of joint algorithm is given in Figure 6.

The advantage of the joint algorithm is to combine the template matching and the BP algorithm and improve the defects of the two algorithms. Using this algorithm, the accuracy of gesture recognition is relatively improved, and the high precision data obtained by Euclidean distance are also a kind of better way to deal with data. The algorithm finally improves the recognition rate of gestures and realizes the effective use of data gloves.

\section{Result and Discussion}

In this paper, the $\mathrm{C}$ language programming software keil5, which is built on the windows environment, was adopted to realize the gesture feature extraction. The template matching recognition algorithm is realized by $\mathrm{C}$ language by $\mathrm{VC}++6.0$. The $\mathrm{BP}$ algorithm realizes the recognition of the nine kinds of gestures by the implementation of MATLAB, which reach the usual recognition rate and comes out the conclusion that the recognition rate was largely influenced by the joint algorithm through the comp 


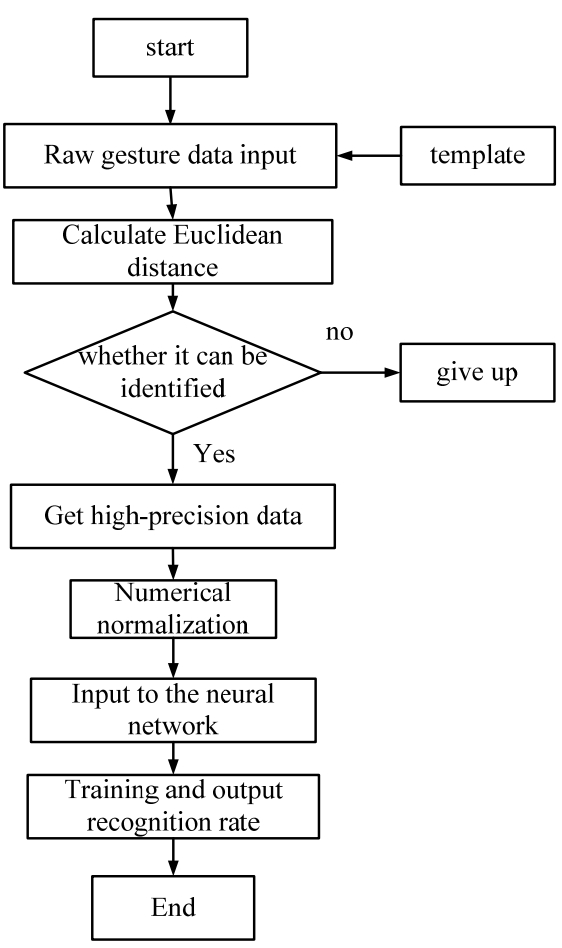

Fig. 6 Joint algorithm flow chart

\subsection{Comparison of BP Algorithm and Joint Algorithm.}

The error drop curve comparison which made by BP algorithm and joint algorithm in the 2000 learning process is shown in Figure 7. It can be seen from the figure that the final error of the BP algorithm after the 1999 training is 0.0040612 . The error of the joint algorithm after 1164 training is 0.00099769. The two are almost an order of magnitude difference, so it can be concluded that the joint algorithm has a faster convergence rate than the BP neural network algorithm [17]. The average training time comparison which made by BP algorithm and joint algorithm in 2000 learning is shown in Table 2. You can see the joint algorithm has faster training time.

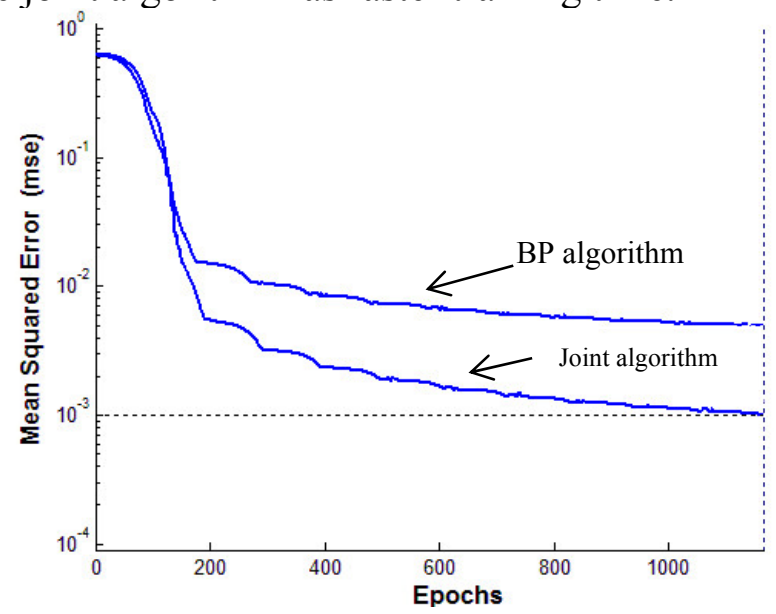

Fig. 7 Error curve comparison chart

Table 2. Average training time comparison

\begin{tabular}{cc}
\hline algorithm & Average training time (S) \\
\hline BP algorithm & 0.0542 \\
Joint algorithm & 0.0134 \\
\hline
\end{tabular}

\subsection{Gestures Comparison of Different Detectors.}

In this paper, we collected the gesture data of five different detectors, and we used the algorithm to compare the gesture recognition rate. The recognition rate was calculated as follows:

$$
\theta=\frac{N_{a}}{N_{b}} \times 100 \%
$$


Where represents the recognition rate, represents the number of successful identification, represents the total number of recognition.

As it can be seen in Table 3, the average recognition rate of joint algorithm for different detectors is significantly higher than that of the other two algorithms, and we see that the gesture data of separate detectors are not identical. For example, the detection rate of detector 2 is relatively low, and the recognition rate of the detector 5 is kept at a high level, which may be due to the fact that the size of the glove during the design of the glove is similar to that of the detector 5, and the data of the detector 5 is kept at a high level during the bending. You can see the joint algorithm for each detector gestures can get a higher recognition rate. And it can solve the problem that different detectors have different recognition rate.

Table 3. Recognition rate comparison

\begin{tabular}{cccc}
\hline Data & Template matching & BP algorithm & Joint algorithm \\
\hline All data & $96.7 \%$ & $98.4 \%$ & $99.8 \%$ \\
Detector 1 & $99.6 \%$ & $100 \%$ & $100 \%$ \\
Detector 2 & $89.3 \%$ & $99 \%$ & $96 \%$ \\
Detector 3 & $95.9 \%$ & $100 \%$ & $100 \%$ \\
Detector 4 & $98.8 \%$ & $99.5 \%$ & $100 \%$ \\
Detector 5 & $100 \%$ & $100 \%$ & $100 \%$ \\
\hline
\end{tabular}

\subsection{Gesture Recognition.}

Built on the above algorithm, this paper uses the visual programming software MATLAB to verify the accuracy of gesture recognition, sends data through the serial port in real time, and tests the gesture according to the MATLAB program. As is illustrated in figure 8, the gesture is basically identifiable, and the gestures we set will be displayed on the image written in MATLAB. The process of Gesture recognition may have some delay phenomenon, mainly because the data transmission time has interval, but does not affect the accuracy of gestures.

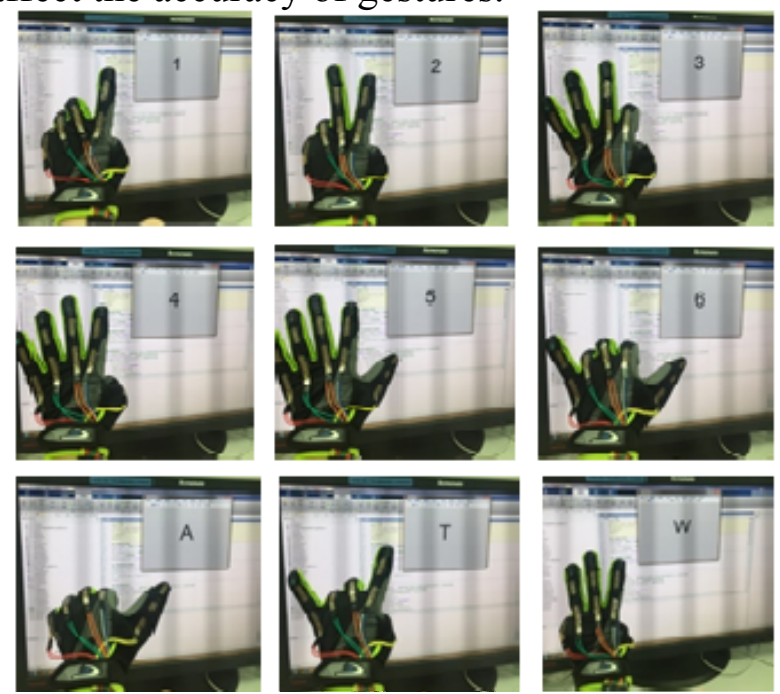

Fig. 8 Real-time gesture recognition display diagram

\section{Conclusion}

In conclusion, we designed the gesture recognition data glove for collection of the gesture data, and built the template matching recognition template from the mean value. The collected identifiable data are normalized and input to the neural network to obtain the recognition rate of the joint algorithm. And the recognition rate of the joint algorithm is compared with the recognition of the template and the recognition rate of the $\mathrm{BP}$ algorithm. The process adopts the single-chip $\mathrm{C}$ language Keil5 to collect gesture data, $\mathrm{VC}++6.0$ to realize the template matching process, MATLAB to realize the neural network, and finally implements a joint algorithm. From the design of data glove, this glove is cheap and easy to improve. More importantly, the high recognition rate could be obtained. 
From the experimental and analytical results, the joint algorithm is a simple identification algorithm which is adaptable and suitable for gesture recognition.

At present, our research on gesture recognition is built on a single background and focuses on the systems that can establish high recognition rates and identify results. But in real life, gesture recognition is a complex process, many environmental factors will affect the gesture recognition effect, in which data gloves are a relatively effective way. The light and other environmental problems will not exert a significant impact on the gesture recognition, but there are many drawbacks for the data gloves because of the fixed pattern. Where after, we can transform the sensor to continue the test, from static gestures to dynamic gestures, constantly trying to find new algorithms, and design more accurate wearable data gloves.

\section{Acknowledgments}

This work was supported by the National Natural Science Foundation of China (NSFC 61504148), the Basic Science and Frontier Technology Research Program of Chongqing (cstc2016jcyjA0315, cstc2017shmsA50001), Chongqing Collaborative Innovation Center for Brain Science, and Youth Innovation Promotion Association of CAS (2016340).

\section{References}

[1]. Lei Li, Dashun QUE. Design of data-glove and Chinese sign language recognition system based on ARM9. 2015 IEEE 12th International Conference on Electronic Measurement \& Instruments. Qingdao, China, 2015, p.1163-1167.

[2]. Qing $\mathrm{Fu}$, Junchen Shen, Xiying Zhang. Gesture recognition with kinect for automated sign language translation. Journal of Beijing Normal University (Natural Science). Vol. 49 (2013) No. 6, p.586-587.

[3]. Lei Lu, Jinling Zhang, Yingjie Zhu, et al. A Static Gesture Recognition Method Based on Data Glove. Journal of Computer-Aided Design \& Computer Graphics. Vol. 27 (2015) No. 12, p.2411-2416.

[4]. Danling Lu, Yuanlong Yu, Huaping Liu. Gesture recognition using data glove: an extreme learning machine method. IEEE International Conference on Robotics \& Biomimetics. Qingdao, China, 2017, p.1349-1354.

[5]. Shihui Zhang, Feifei Zhou, Shunchao Guo. Gesture recognition based on feature combination and partial least squares dimensionality reduction. Journal of Yanshan University. Vol. 38 (2014) No. 1, p.41-47.

[6]. Dongjie Li, Junxiang Li, Yue Zhang, et al. Gesture recognition of data glove based on PSO-Improved BP neural network. Electri c Machines and Control. Vol. 18 (2014) No. 8, p.88-92.

[7]. Zhiquan Feng, Yan Jiang. A Survey of Hand Gesture Recognition. JOURNAL OF UNIVERSITY OF JINAN (Sci.\& Tech.). Vol. 27 (2013) No. 4, p.337-339.

[8]. Hugo Jair Escalante, Eduardo F. Morales, L. Enrique Sucar. A naïve Bayes baseline for early gesture recognition. Pattern Recognition Letters. Vol. 73 (2016) No. C, p.91-99.

[9]. Jun Xu, Yinglong Dong, Yanan Tang. Gesture Recognition Based on Wearable Sensing. The 30th Chinese Control and Decision Conference. Yinchuan, China, 2016, p.36-41.

[10]. Jiali Cui, Wei Xie, Yiding Wang, et al. Static Gesture Digit Recognition Based on Shape Features. Journal of North China University of Technology. Vol. 27 (2015) No. 3, p.43-44. 
[11]. Zhiming Li, Yong Tan, Shiming Xu. STM32 Embedded System Development Practical Guide. China Machine Press, 2013, p.11-40.

[12]. Zhen Yuan, Man Yuan. The Noise Reduction of Data Acquisition Circuit. Journal of Chongqing Institute of Technology. Vol. 19 (2005) No. 3, p.32-34.

[13]. Haipeng Wang, Shuyi Jia, Ziling Wang. Multi-sensor Formation Targets Template Matching Tracking Algorithm. 2016 International Conference on Mechatronics. Bangkok, 2016, p.149-154.

[14]. Jingguo Yi, Jianghua Cheng, Xishu Ku. Review of Gestures Recognition Based on Vision. Computer Science. Vol. 43 (2016) No.6A, p.103-106.

[15]. SimonHaykin. Neural Networks. China Machine Press, 2004, p.5-35.

[16]. Na Wei. The application of template matching and BP neural network in ship identification. SHIP SCIENCE AND TECHNOLOGY. Vol. 38 (2016) No.10A, p.134-135.

[17]. Fengye Hu, Lu Wang, Shanshan Wang, et al. A Human Body Posture Recognition Algorithm Based on BP Neural Network for Wireless Body Area Networks. China Communications. Vol. 13 (2016) No.8, p.198-208. 